Published in final edited form as:

Curr Opin Hematol. 2008 November ; 15(6): 549-554. doi:10.1097/MOH.0b013e328311891f.

\title{
New Advances in Hematopoietic Cell Transplantation
}

\author{
Effie W. Petersdorf, MD and \\ Division of Clinical Research, D4-100, Fred Huchinson Cancer Research Center, 1100 Fairview \\ Ave N, Seattle, WA USA 98109, epetersd@fhcrc.org
}

John A. Hansen, MD

Division of Clinical Research, D2-100, Fred Huchinson Cancer Research Center, 1100 Fairview

Ave N, Seattle, WA USA 98109, jhansen@fhcrc.org, 206-667-5111

\section{Abstract}

Purpose of the review-This review highlights sentinel work published since 2006 on the definition of the transplantation barrier and the elucidation of cytokine and immune response gene variation in defining post-transplant risks.

Recent findings-Recent work has defined the relative importance of matching for the classical HLA-A, B, C, DRB1, DQB1 genes, and the importance of additive effects of multi-locus disparity. This work provides a new framework for donor identification and extends the use of single locus HLA-DQB1 mismatched donors without compromising the success of the transplant. New data demonstrate that permissible class I mismatches may be defined by donor-recipient mismatching at certain residues. The concept that the extended HLA haplotype carries undetected but functional variation, provides an approach for mapping novel transplantation determinants, and a means to further improve the clinical results of transplantation from HLA matched unrelated donors. Finally, the role of sequence variation in immune response and cytokine genes provides a means for assessing risks for a given transplant recipient and may aid in the planning of the transplant procedure.

Summary-Optimizing the results of unrelated donor transplantation requires an understanding of risks associated with variation of HLA genes within the major histocompatibility complex (MHC), and of genes that participate in the immune response and inflammatory pathways.

\section{Keywords}

HLA; microsatellites; haplotypes; cytokine genes; immune response genes

\section{Introduction}

Major advances in the field of immunogenetics have contributed to the success of allogeneic hematopoietic cell transplantation (HCT) as a curative modality. The availability of DNAbased methods for typing the highly polymorphic HLA genes have provided new information regarding the functional consequences of allelic variation. Currently, the standard for the selection of unrelated donors includes high resolution typing and matching for HLA-A, B, C, DRB1 and DQB1 alleles. Although allele identity between the transplant recipient and unrelated donor can lower the risks of clinically significant acute GVHD, chronic GVHD, and mortality, matching for HLA genes does not guarantee that these complications will not occur. Clinical GVHD can be observed in as high as $60 \%$ of wellmatched transplant recipients depending on the immunosuppressive regimen. Recent evidence strongly suggests that genetic variation within the major histocompatibility complex (MHC) residing outside of the coding regions of the classical HLA genes can contribute to increased post-transplant risks even after HLA matched transplantation. 
Furthermore, polymorphism in cytokine genes and immune response genes play an important role in modulating the effects of tissue injury that constitute the GVHD syndrome.

\section{The MHC Region}

In this section we will present new data on locus-specific risks and the significance of extended MHC haplotypes.

\section{Matching for Classical HLA Genes}

Donor-recipient matching for the classical HLA genetic loci, HLA-A, B, C, DRB1 and DQB1 has served as the cornerstone of unrelated HCT. Although the importance of the HLA system in transplantation has been well-defined for several decades, investigation into the functional significance of sequence polymorphism of class I and II genes has been feasible since the late 1980s when polymerase chain reaction (PCR) technology became available. Since then, the clinical significance of donor matching has been defined. [1-9]

Recently, an analysis of a large cohort of unrelated donor transplants facilitated by the National Marrow Donor Program (NMDP) has shed new light on the relative importance of matching for HLA genes. [1] A total of 3860 patients and their unrelated donors were evaluated for locus-specific risks. When compared to patients matched at all 5 HLA-A, B, C, DRB1, DQB1 loci ("10/10" matched), the presence of a single mismatch was associated with adverse outcome, with the exception of single HLA-DQB1 locus mismatches. These results indicate that a single HLA-DQ mismatch is better tolerated than HLA-A, B, C or DRB1 mismatches; when no matched donor can be identified, then use of a donor with a single HLA-DQ mismatch might be acceptable. Transplantation from HLA-A, B, C, DRB1 matched donors (" $8 / 8$ " matched) yields outcomes equivalent to that after 10/10 matching. Mismatching for two or more determinants, however, was associated with increased risk of acute GVHD and mortality. HLA-DQB1 disparity was detrimental when mismatching at other HLA loci was also present; therefore, when a donor is already known to have one HLA-A, B, C, or DRB1 mismatch, prospective HLA-DQB1 typing may help to define the total number of mismatches.

\section{Permissible HLA Mismatches}

The concept that alloreactivity could be mapped to discrete residues of the HLA molecule which participate in defining peptide binding or direct contact with the T cell receptor, was initially shown by Ferrera et al. [10] In this study, risks associated with donor-recipient amino acid mismatching at key residues of the HLA-B molecule were measured. Disparity at residue 116 was associated with increased risk of clinically significant acute GVHD and transplant-related mortality (TRM) compared to matching at this residue.

Extension of the Ferrara observations were recently made by the Japan Marrow Donor Program (JMDP) in 4866 unrelated donor-recipient pairs. [11] Donor-recipient disparity for Tyr9 - Phe9 of HLA-A and Tyr9 - Ser9, Asn77 - Ser77, Lys80 - Asn80, Tyr99 - Phe99, Leu116 - Ser116, and Arg156 - Leu156 of HLA-C were each associated with significantly increased risks of severe acute GVHD. A similar theme is emerging for epitopes encoded by HLA-DP. $[9,12]$ These studies demonstrate the importance of correlating structure with function as a clinically relevant tool for donor selection.

\section{HLA Haplotypes}

High resolution typing methods for unrelated donor matching provides a surrogate for the haplotype matching that is feasible between genotypically identical siblings. Even though unrelated donors and recipients may share the same HLA alleles, the alleles may be encoded 
on different haplotypes. The concept that the haplotype may define a series of markers, some detected, others undetected, which can be used to map functionally significant variation, has been applied in many models. Substantial information is currently available on the extensive sequence variation encoded within the MHC and its organization on haplotypes. [13] The complete sequencing of several common European haplotypes demonstrates that the full extent of MHC region variation has not yet reached a plateau. These data strongly suggest that haplotype-based approaches are needed for fine mapping of functional variation. Since the MHC harbors regions of high linkage disequilbrium (LD), haplotype-based approaches can serve as powerful tools for identifying variation that cause disease. [14-17]

To test the hypothesis that novel undetected MHC resident variation encoded on HLA haplotypes could be responsible for post-transplant risks after HLA allele matched unrelated donor transplantation, a novel method for phasing HLA alleles has recently been developed to define the physical linkage of HLA-A, B and DRB1 alleles. [18] Given that HLA-B maps $1.4 \mathrm{Mb}$ centromeric to HLA-A and 1.2 Mb telomeric to HLA-DRB1, HLA-B was used as a point of separation for the two haplotypes using arrays of HLA-B-specific oligonucleotide probes. Application of the phasing method to 10/10 allele-matched unrelated donor-recipient pairs uncovered a $20 \%$ frequency of haplotype mismatching which was associated with significantly increased risk of clinically severe acute GVHD. [19]

These results suggest that untyped variation carried on the HLA haplotype might cause GVHD after HLA matched unrelated HCT, either from donor-recipient mismatching and/or from the direct effects of the variation. Evidence to support a role for haplotype-associated variation has recently been established in two studies that have employed microsatellite markers as a mapping tool. [20,21] In a study of Japanese patients, polymorphism of the tumor necrosis factor (TNF) complex residing in the class III region of the MHC correlated with lower survival among patients who developed GVHD. [20] The identification of functional MHC variation in the class I, II and III regions has been observed in a large retrospective analysis of 10/10 matched donor-recipient pairs of Caucasian background. [21]

\section{Practical applications}

Haplotypes have been used to define optimal unrelated donor registry size and composition, and to predict the likelihood that a potential unrelated donor typed at low resolution for HLA-A, B, DR loci, will be allele matched at HLA-A, B,C, DRB1 and DQB1. The use of haplotype probabilities may increase the efficiency of an unrelated donor search and aid in prioritizing confirmatory typing of donors who are most likely allele matched with the recipient. To meet these needs, statistical methods have been developed to infer haplotypes when family data is not available, as in the case of unrelated donors. Application of haplotype inference methods to unrelated donor registry data must be robust enough to accommodate incomplete HLA genotype information, or variable levels of resolution of HLA alleles. [22-25] Due to the strong positive, long-range LD within the MHC, knowledge of the three-locus HLA-A, B, DR haplotype is descriptive of higher resolution definition of the extended haplotype. [23]

\section{Use of haplotypes for recruitment of unrelated donors}

To meet the needs of patients initiating a search for an unrelated donor, registries must have donors with both common and unique phenotypes. Several approaches for donor recruitment have been taken, including minority recruitment to increase the HLA diversity and to increase the probability that patients with uncommon phenotypes will identify suitable donors. [26] A novel approach for donor recruitment has recently been described in a study by the DKMS German Bone Marrow Donor Center. [27] This study demonstrates that it is 
feasible to increase the diversity of a donor registry by recruiting the relatives of registered donors who have rare HLA phenotypes. In this way, the proportion of donors with uncommon phenotypes can be successfully recruited.

\section{Non-MHC Genetic Factors Affecting Transplant Outcome}

In this section we will summarize new information on the clinical significance of cytokine and immune response gene variation and GVHD.

\section{Genetic variants encoding non-MHC transplant determinants (minor histocompatibility antigens)}

Non-MHC polymorphisms occurring throughout the genome encode transplant determinants known as minor histocompatibility antigens (mHA). [28] GVHD in HLA identical sibling donor (MRD) HCT is attributable to mHA antigens. Since MRD pairs share 50\% of their genomes and minimal sharing occurs between URD pairs, disparity for mHA and the risk of GVHD must be greater in the latter. [29] Although accounting quantitatively for mHA disparity could greatly facilitate donor selection, there currently is no technology available capable of measuring the total mHA burden for any given transplant pair.

\section{Genetic variants affecting the function of immune response genes (IRG)}

In addition to genetic diversity that causes disparity for mHA, there is in every individual extensive polymorphism that determines gene function and controls phenotype. This variation includes single nucleotide polymorphisms (SNPs) and structural differences known as copy number variation (CNV) polymorphisms. The majority of genetic variation has no functional significance, however selected SNPs and CNV and short tandem repeats (microsatellite) can serve as useful markers for functional variants because of the significant linkage disequilibrium that occurs across distances as long as several hundred kilobases $(\mathrm{KB})$.

Genetic variation affects function by regulating transcription and alternative exon splicing, and encoding critical amino acid substitutions. Through various mechanisms, these functional polymorphisms control IRG by regulating the activity immune cells, receptors and cytokines, and by modulating the strength of the inflammatory response. Functional variation can also affect immunity involved in resistance to bacterial, fungal and viral disease, as well as other pathways impacting HCT outcome such as drug metabolism and the toxicity of cytotoxic therapy.

\section{IRG polymorphisms associated with acute GVHD and transplant-related mortality-- The proinflammatory cytokine TNF}

The first reports suggesting that non-MHC polymorphisms might affect HCT outcome utilized microsatellites markers linked to candidate IRG known to play important roles in modulating the alloimmune response. Middleton et al in 1998 reported results of an analysis of TNFd, a dinucleotide (GA) microsatellite located within the tumor necrosis factor (TNF) gene complex, in 49 MRD HCT and found an association with acute GVHD. [30] In a follow-up study, the same group further demonstrated an association of the TNFd3 allele with TRM. [31] However, subsequent studies of TNF promoter region SNPs by Socie et al [32] and Lin et al [33] in cohorts of 100 and 570 MRD cases respectively found no association with acute GVHD or TRM while Bogunia-Kublink et al reported an association of TNFA and TNFB genotypes with toxicity but not GVHD. [34] A study of a SNP mapping to an intron in the TNF gene in $160 \mathrm{MRD}$ transplants by Mullighan et al reported associations with acute and chronic GVHD but not TRM. [35] Keen et al studied TNF in 182 URD cases and found an association with TRM but not GVHD. [36] 


\section{Studies of the regulatory cytokine IL-10}

The first published study of IL-10 variation in HCT, by Middleton et al, reported the association of a microsatellite polymorphism, IL-10G, located at position -1064 in the promoter region of the IL10 gene of the patient with acute GVHD in MRD cases. [30] This association was reinforced in a follow-up study by the same group in 144 HCT cases. [37] Takahashi et al analyzed the IL10G microsatellite in 62 HCT cases and found an association of high repeat numbers ( $>13$ alleles) in the donor with chronic but not acute GVHD. [38] Rocha et al studied 107 MRD cases and found an association of the IL10G microsatellite in the patient with chronic GVHD, but not with acute GVHD or TRM. [39] Overall, associations of IL10 promoter region variation with GVHD or TRM has been demonstrated in at least ten different studies. [37] [38] [32] [39] [33] [36] [35] [40] [41] [42] Lin et al reported a two phase discovery and validation analysis of four promoter region SNPs. [33] Significant associations with acute GVHD and TRM were found for SNPs -592 and -1082 in the patient in the discovery cohort of 570 MRD cases, and this was confirmed in a second independent cohort of 423 MRD cases. An analysis of the combined cohorts $(n=993)$ showed that the patients' IL10/-592*A/A genotype was associated with a decreased risk of grades III-IV GVHD and TRM compared to the IL10/-592*C/C genotype. [33] Mullighan et al [35] and Kim et al [41] found no association of IL10 genotypes with chronic, but not acute GVHD. In a study of 182 URD HCT, Keen et al reported an association of IL10 promoter variation in the donor with TRM but not with GVHD. [36] Bettens et al analyzed the IL10G microsatellite in 131 URD HCT and found an association of the low repeat variants ( $<12$ alleles) in the patient with better survival. [42] In a study of 682 unrelated donor HCT cases at our center, however, we have found no association of 4 IL10 promoter region SNPs in either patient or donor with acute GVHD or TRM (unpublished data).

Lin et al extended the analysis of the IL10 pathway by examining a coding SNP in the IL10RB gene at cDNA position $238(A / G)$. [43] The $c 238^{*} G$ allele of the donor was significantly associated with a lower risk of acute GVHD and provided protection among recipients with the high-risk IL10/-592*A/C or $A A$ genotypes but not among those with the IL10/-592*C/C genotype, suggesting an interaction between the donor IL10RB/c238 and recipient IL10/-592 genotypes.

\section{Summary of IRG association studies with GVHD}

The data reviewed above, although representing only 2 of the prominent candidate IRG genes, TNF and IL10, illustrates the overall problematic. Similar data suggesting associations of several other IRG genes with GVHD and survival including CTLA4, IFNG, IL-1, IL-1RA, IL-2, IL-6, IL-7R, NOD2 and TNFRII have been reported, however these results like those for TNF and IL10 are often inconsistent or lack rigorously designed validation studies. [30] [37] [32] [38] [31] [44] [39] [33] [45] [46] [47] [35] [48] [40] [43] [36] [40] [49] [34] [50] [42] [51] [52] [53] Three recent review articles have also addressed the integration and interpretation of these several different studies. [54] [55] Unfortunately, it is not possible with the cumulative data currently available to clearly distinguish true positive associations from false positive or false negative findings. There may be several factors contributing to this confusing situation. The most likely explanations include: 1) heterogeneity between patient populations (and differences due to unknown patient and disease risk factors such as diagnosis, prior therapy and disease stage, transplant protocols and possibly population differences or population admixture); 2) the markers selected may be in weal linkage disequilibrium with the relevant functional variant; and 3) low sample size and lack of sufficient statistical power. The latter is likely to be the primary reason for lack of sensitivity and the occurrence of false positive associations. Results from most studies are based on sample sizes of only a few hundred patients. By contrast, genetic risk 
studies of common immune mediated diseases such as type 1 diabetes have required samples of several thousand patients.

\section{Future studies of genetic factors affecting GVHD}

Future studies IRG associations with GVHD and related complications and mortality will need to carefully address basic study design questions such the optimal study population, power considerations and the scope of the genetic analysis. Mullally and Ritz, [28] and Mullighan and Brady, [29] have recently outlined the emerging technologies available for performing whole genome scans and the impact that these new approaches can have on the discovery of genes and pathways, many of which may be unknown, that critically control the strength of GVHD, HCT-related toxicity and survival. This knowledge could have great utility for predicting risk, counseling patients, guiding donor selection and the choice of the choice of alternative transplant procedure. [56] The potential for bringing personal medicine to the HCT clinic requires a comprehensive description of all the functional genetic determinants associated with GVHD and mortality, and an understanding of how these genetic factors interact with the other clinical covariables that affect HCT outcome.

\section{Conclusion}

Genetic diversity of HLA and IRGs has functional implications in unrelated donor HCT. Sequence disparity between the transplant recipient and donor for the classical class I and II genes is associated with risks of graft rejection, GVHD and mortality. Polymorphism of IRGs modulates the strength of the inflammatory response after HCT. Optimizing unrelated donor transplantation includes consideration of the HLA match status of the recipient and donor, and avoidance of high-risk HLA mismatches. Future advances in HCT may include the incorporation of information on recipient and donor IRG variation in risk assessment and planning of the transplant procedure.

\section{Acknowledgments}

This work was supported by grants from the National Institutes of Health AI33484 (JAH, EWP), CA18029 (JAH,EWP), HL087690 (JAH) and CA100019 (EWP).

\section{References}

1. Lee SJ, Klein J, Haagenson M, et al. High-resolution donor-recipient HLA matching contributes to the success of unrelated donor marrow transplantation. Blood. 2007; 110:4576-4583. [PubMed: 17785583] This study represents the largest analysis of unrelated donor transplants to date. New findings that single HLA-DQB1 allele mismatches may be tolerated, provides a means to extend HCT to DQB1-mismatched donors when matched donors are not available.

2. Petersdorf EW, Anasetti C, Martin PJ, et al. Limits of HLA mismatching in unrelated hematopoietic cell transplantation. Blood. 2004; 104:2976-2980. [PubMed: 15251989]

3. Flomenberg N, Baxter-Lowe LA, Confer D, et al. Impact of HLA class I and class II high resolution matching on outcomes of unrelated donor bone marrow transplantation: HLA-C mismatching is associated with a strong adverse effect on transplant outcome. Blood. 2004; 104:1923-1930. [PubMed: 15191952]

4. Chalandon Y, Tiercy JM, Schanz U, et al. Impact of high-resolution matching in allogeneic unrelated donor stem cell transplantation in Switzerland. Bone Marrow Transplant. 2006; 37:909916. [PubMed: 16565739]

5. Yakoub-Agha I, Mesnil F, Kuentz M, et al. Allogeneic marrow stem-cell transplantation from human leukocyte antigen-identical siblings versus human leukocyte antigen-allelic-matched unrelated donors (10/10) in patients with standard-risk hematologic malignancy: a prospective study from the French Society of Bone Marrow Transplantation and Cell Therapy. J Clin Oncol. 2006; 24:5695-5702. [PubMed: 17116940] 
6. Hows JM, Passweg JR, Tichelli A, et al. Comparison of long-term outcomes after allogeneic hematopoietic stem cell transplantation from matched sibling and unrelated donors. Bone Marrow Transplant. 2006; 38:799-805. [PubMed: 17075568]

7. Kennedy-Nasser AA, Leung KS, Mahajan A, et al. Comparable outcomes of matched-related and alternative donor stem cell transplantation for pediatric severe aplastic anemia. Biol Blood Marrow Transplant. 2006; 12:1277-1284. [PubMed: 17162209]

8. Morishima Y, Sasazuki T, Inoko H, et al. The clinical significance of human leukocyte antigen (HLA) allele compatibility in patients receiving a marrow transplant from serologically HLA-A, HLA-B, and HLA-DR matched unrelated donors. Blood. 2002; 99:4200-4206. [PubMed: 12010826]

9. Morishima Y, Yabe T, Matsuo K, et al. Effects of HLA allele and killer immunoglobulin-like receptor ligand matching on clinical outcome in leukemia patients undergoing transplantation with T-cell-replete marrow from an unrelated donor. Biol Blood Marrow Transplant. 2007; 13:315-328. [PubMed: 17317585]

10. Ferrara GB, Bacigalupo A, Lamparelli T, et al. Bone marrow transplantation from unrelated donors: the impact of mismatches with substitutions at position 116 of the human leukocyte antigen class I heavy chain. Blood. 2001; 98:3150-3155. [PubMed: 11698304]

11. Kawase T, Morishima Y, Matsuo K, et al. High-risk HLA allele mismatch combinations responsible for severe acute graft versus host disease and implication for its molecular mechanism. Blood. 2007; 110:2235-2241. [PubMed: 17554059] The functional importance of donor-recipient mismatching for key polymorphic class I residues extends knowledge on the nature of epitopes involved in peptide-binding repertoire and contact with the $\mathrm{T}$ cell receptor important in defining GVHD risk.

12. Fleischhauer K, Locatelli F, Zecca M, et al. Graft rejection after unrelated donor hematopoietic stem cell transplantation for thalassemia is associated with nonpermissive HLA-DPB1 disparity in host-versus-graft direction. Blood. 2006; 107:2984-2992. [PubMed: 16317094]

13. Horton R, Gibson R, Coggill P, et al. Variation analysis and gene annotation of eight MHC haplotypes: the MHC Haplotype Project. Immunogenetics. 2008; 60:1-18. [PubMed: 18193213] A comprehensive examination of common human HLA haplotypes provides a unprecedented view of the sequence variation within the MHC.

14. Traherne JA, Horton R, Roberts AN, et al. Genetic analysis of completely sequenced diseaseassociated MHC haplotypes identifies shuffling of segments in recent human history. PLoS Genetics. 2006; 2:e9. [PubMed: 16440057]

15. Smith WP, Vu Q, Li SS, et al. Toward understanding MHC disease associations: partial resequencing of 46 distinct HLA haplotypes. Genomics. 2006; 87:561-571. [PubMed: 16434165]

16. Miretti MM, Walsh EC, Ke X, et al. A high-resolution linkage-disequilibrium map of the human major histocompatibility complex and first generation of tag single-nucleotide polymorphisms. Am J Hum Genet. 2005; 76:634-646. [PubMed: 15747258]

17. de Bakker PI, McVean G, Sabeti PC, et al. A high-resolution HLA and SNP haplotype map for disease association studies in the extended human MHC. Nat Genet. 2006; 38:1166-1172. [PubMed: 16998491]

18. Guo Z, Hood L, Malkki M, Petersdorf EW. Long-range multilocus haplotype phasing of the MHC [erratum appears in Proc Natl Acad Sci U S A. 2006 Jun 13;103(24):9374]. PNAS. 2006; 103:6964-6969. [PubMed: 16632595]

19. Petersdorf EW, Malkki M, Gooley TA, et al. MHC haplotype matching for unrelated hematopoietic cell transplantation. PLoS Medicine. 2007; 4:e8. [PubMed: 17378697] A longrange phasing technique was applied to define the clinical significance of extended HLA haplotypes in unrelated donor HCT. This study demonstrates that undetected MHC region variation may increase the risk of acute GVHD. These results provide a novel approach for mapping genes associated with transplant outcomes.

20. Li S, Kawata H, Katsuyama Y, et al. Association of polymorphic MHC microsatellites with GVHD, survival, and leukemia relapse in unrelated hematopoietic stem cell transplant donor/ recipient pairs matched at five HLA loci. Tissue Antigens. 2004; 63:362-368. [PubMed: 15009808] 
21. Malkki M, Gooley TA, Horowitz MM, et al. Mapping MHC-resident transplantation determinants. Biol Blood Marrow Transplant. 2007; 13:986-995. [PubMed: 17640603]

22. Gourraud PA, Lamiraux P, El Kadhi N, et al. Inferred HLA haplotype information for donors from hematopoietic stem cells donor registries. Hum Immunol. 2005; 66:563-570. [PubMed: 15935894]

23. Maiers M, Gragert L, Klitz W. High-resolution HLA alleles and haplotypes in the United States population. Hum Immunol. 2007; 68:779-788. [PubMed: 17869653] This analysis provides a comprehensive view of human HLA haplotypes and serves as an important resource for HLA research.

24. Kollman C, Maiers M, Gragert L, et al. Estimation of HLA-A, -B, -DRB1 haplotype frequencies using mixed resolution data from a national registry with selective retyping of volunteers. Hum Immunol. 2007; 68:950-958. [PubMed: 18191722]

25. Muller CR, Ehninger G, Goldmann SF. Gene and haplotype frequencies for the loci hLA-A, hLAB, and hLA-DR based on over 13,000 German blood donors. Hum Immunol. 2003; 64:137-151. [PubMed: 12507825]

26. Confer DL. The National Marrow Donor Program. Meeting the needs of the medically underserved. Cancer. 2001; 91 Suppl. 1:274-278. [PubMed: 11148593]

27. Schmidt AH, Stahr A, Baier D, et al. Selective recruitment of stem cell donors with rare human leukocyte antigen phenotypes. Bone Marrow Transplant. 2007; 40:823-830. [PubMed: 17724442] Recruitment of the family members of registered unrelated donors with rare alleles and haplotypes provides a means to increase the pool of rare haplotypes in a donor registry. This study by the DKMS registry demonstrates a novel approach for increasing the genetic diversity of a donor pool.

28. Perreault C, Décary F, Brochu S, et al. Minor histocompatibility antigens. Blood. 1990; 76:12691280. [PubMed: 2207305]

29. Martin PJ. Increased disparity for minor histocompatibility antigens as a potential cause of increased GVHD risk in marrow transplantation from unrelated donors compared with related donors. Bone Marrow Transplant. 1991; 8:217-223. [PubMed: 1958902]

30. Middleton PG, Taylor PRA, Jackson G, et al. Cytokine gene polymorphisms associating with severe acute graft-versus-host disease in HLA-identical sibling transplants. Blood. 1998; 92:39433948. [PubMed: 9808588]

31. Cavet J, Dickinson AM, Norden J, et al. Interferon-gamma and interleukin-6 gene polymorphisms associate with graft-versus-host disease in HLA-matched sibling bone marrow transplantation. Blood. 2001; 98:1594-1600. [PubMed: 11520812]

32. Socie G, Loiseau P, Tamouza R, et al. Both genetic and clinical factors predict the development of graft-versus-host disease after allogeneic hematopoietic stem cell transplantation. Transplantation. 2001; 72:699-706. [PubMed: 11544434]

33. Lin M-T, Storer B, Martin PJ, et al. Relation of an interleukin-10 promoter polymorphism to graftversus-host disease and survival after hematopoietic-cell transplantation. N Engl J Med. 2003; 349:2201-2210. [PubMed: 14657427]

34. Bogunia-Kubik K, Polak M, Lange A. TNF polymorphisms are associated with toxic but not with aGVHD complications in the recipients of allogeneic sibling haematopoietic stem cell transplantation. Bone Marrow Transplant. 2003; 32:617-622. [PubMed: 12953135]

35. Mullighan C, Heatley S, Doherty K, et al. Non-HLA immunogenetic polymorphisms and the risk of complications after allogeneic hemopoietic stem-cell transplantation. Transplantation. 2004; 27:587-596. [PubMed: 15084940]

36. Keen LJ, Defor TE, Bidwell JL, et al. Interleukin-10 and tumor necrosis factor alpha region haplotypes predict transplant-related mortality after unrelated donor stem cell transplantation. Blood. 2004; 103:3599-3602. [PubMed: 14701704]

37. Cavet J, Middleton PG, Segall M, et al. Recipient tumor necrosis factor-alpha and interleukin-10 gene polymorphisms associate with early mortality and acute graft-versus-host disease severity in HLA-matched sibling bone marrow transplants. Blood. 1999; 94:3941-3946. [PubMed: 10572111] 
38. Takahashi H, Furukawa T, Hashimoto S, et al. Contribution of TNF-alpha and IL-10 gene polymorphisms to graft-versus-host disease following allohematopoietic stem cell transplantation. Bone Marrow Transplant. 2000; 26:1317-1323. [PubMed: 11223972]

39. Rocha V, Franco RF, Porcher R, et al. Host defense and inflammatory gene polymorphisms are associated with outcomes after HLA-identical sibling bone marrow transplantation. Blood. 2002; 100:3908-3918. [PubMed: 12393699]

40. Karabon L, Wysoczanska B, Bogunia-Kubik K, et al. IL-6 and IL-10 promoter gene polymorphisms of patients and donors of allogeneic sibling hematopoietic stem cell transplants associate with the risk of acute graft-versus-host disease. Hum Immunol. 2005; 66:700-710. [PubMed: 15993715]

41. Kim DH, Lee NY, Sohn SK, et al. IL-10 promoter gene polymorphism associated with the occurrence of chronic GVHD and its clinical course during systemic immunosuppressive treatment for chronic GVHD after allogeneic peripheral blood stem cell transplantation. Transplantation. 2005; 79:1615-1622. [PubMed: 15940053]

42. Bettens F, Passweg J, Gratwohl A, et al. Association of TNFd and IL-10 polymorphisms with mortality in unrelated hematopoietic stem cell transplantation. Transplantation. 2006; 81:12611267. [PubMed: 16699452]

43. Lin M-T, Storer B, Martin PJ. Genetic variation in the IL-10 pathway modulates severity of acute graft-versus-host disease following hematopoietic cell transplantation: synergism between IL-10 genotype of patient and IL-10 receptor b genotype of donor. Blood. 2005; 106:3995-4001. [PubMed: 16109775]

44. Cullup H, Dickinson AM, Jackson GH, et al. Donor interleukin 1 receptor antagonist genotype associated with acute graft-versus-host disease in human leucocyte antigen-matched sibling allogeneic transplants. Br J Haematol. 2001; 113:807-813. [PubMed: 11380474]

45. MacMillan ML, Radloff GA, Kiffmeyer WR, et al. High-producer interleukin-2 genotype increases risk for acute graft-versus-host disease after unrelated donor bone marrow transplantation. Transplantation. 2003; 76:1758-1762. [PubMed: 14688528]

46. MacMillan ML, Radloff GA, Defor TE, et al. Interleukin-1 genotype and outcome of unrelated donor bone marrow transplantation. Br J Haematol. 2003; 121:597-604. [PubMed: 12752101]

47. Stark GL, Dickinson AM, Jackson GH, et al. Tumour necrosis factor receptor type II 196M/R genotype correlates with circulating soluble receptor levels in normal subjects and with graftversus-host disease after sibling allogeneic bone marrow transplantation. Transplantation. 2003; 76:1742-1749. [PubMed: 14688526]

48. Holler E, Rogler G, Herfarth H, et al. Both donor and recipient NOD2/CARD15 mutations associate with transplant-related mortality and GvHD following allogeneic stem cell transplantation. Blood. 2004; 104:889-894. [PubMed: 15090455]

49. Seo KW, Kim DH, Sohn SK, et al. Protective role of interleukin-10 promoter gene polymorphism in the pathogenesis of invasive pulmonary aspergillosis after allogeneic stem cell transplantation. Bone Marrow Transplant. 2005; 36:1089-1095. [PubMed: 16247433]

50. Bogunia-Kubik K, Mlynarczewska A, Jaskula E, Lange A. The presence of IFNG 3/3 genotype in the recipient associates with increased risk for Epstein-Barr virus reactivation after allogeneic haematopoietic stem cell transplantation. Br J Haematol. 2006; 132:326-332. [PubMed: 16409297]

51. Holler E, Rogler G, Brenmoehl J, et al. Prognostic significance of NOD2/CARD15 variants in HLA-identical sibling hematopoietic stem cell transplantation: Effect on long term outcome is confirmed in 2 independent cohorts and may be modulated by the type of gastrointestinal decontamination. Blood. 2006; 107:4189-4193. [PubMed: 16424393]

52. Shamim Z, Ryder LP, Heilmann C, et al. Genetic polymorphisms in the genes encoding human interleukin-7 receptor-alpha: prognostic significance in allogeneic stem cell transplantation. Bone Marrow Transplant. 2006; 37:485-491. [PubMed: 16435014]

53. Perez-Garcia A, de la CR, Roman-Gomez J, et al. CTLA-4 polymorphisms and clinical outcome after allogeneic stem cell transplantation from HLA-identical sibling donors. Blood. 2007; 110:461-467. [PubMed: 17384200] 
54. Mullally A, Ritz J, et al. Beyond HLA: the significance of genomic variation for allogeneic hematopoietic stem cell transplantation. Blood. 2007; 109:1355-1362. [PubMed: 17008540] The authors illustrate how emerging technologies are rapidly expanding knowledge of the human genome, and how structural variation and deletions can uniquely contribute to disparity for minor histocompatibility antigens.

55. Mullighan CG, Bardy PG. New directions in the genomics of allogeneic hematopoietic stem cell transplantation. Biol Blood Marrow Transplant. 2007; 13:127-144. [PubMed: 17241919] This recent review provides a critical overview, that goes beyond the scope of this Current Opinion article, and comprehensively summarizes known mHA's, and non-MHC IRG variants, that have been reported associated with HCT outcome.

56. Dickinson AM. Risk assessment in haematopoietic stem cell transplantation: pre-transplant patient and donor factors: non-HLA genetics (Review). Bailliere's Best Practice in Clinical Haematology. 2007; 20:189-207. 